\title{
Apoyo familiar en mayores institucionalizados
}

\author{
$\mathrm{M}^{\mathrm{a}}$ del Mar Molero ${ }^{1}, \mathrm{M}^{\mathrm{a}}$ del Carmen Pérez-Fuentes ${ }^{1}$, José J. \\ Gázquez $^{1}$ y Erica Sclavo ${ }^{2}$ \\ ${ }^{1}$ Universidad de Almería (España); ${ }^{2}$ Universidad de Torino (Italia)
}

\begin{abstract}
Los cambios sociales, eventos vitales y dificultades que acompañan al envejecimiento, pueden declinar en situaciones de soledad, y en el peor de los casos, de aislamiento. En casos de institucionalización, el efecto atenuante que tiene el apoyo familiar, puede verse incrementado ante la necesidad de hacer frente a una nueva etapa como la vejez. El objetivo de este estudio es analizar la frecuencia y el tipo de contactos familiares que mantienen en residencia, así como su grado de satisfacción al respecto. Todo ello, en una muestra de 50 personas mayores de 70 años institucionalizados, para lo cual se aplicó el Cuestionario de Evaluación de Calidad de Vida en contexto Residencial "CECAVI-R". Así, los resultados muestran que los mayores, de edad más avanzadas, son quienes con mayor frecuencia, mantienen contactos familiares desde la residencia. Teniendo en cuenta el género, la frecuencia en los contactos familiares variaba en función de la persona que visitaba (nietos, hermanos, etc.). Y por último, el análisis de la satisfacción hacia las relaciones familiares es positivo, mostrándose los residentes más jóvenes como los menos satisfechos.
\end{abstract}

Palabras clave: Redes de apoyo. Familia. Mayores. Institucionalización. Satisfacción.

Family support in older institutionalized people. Social changes, life events, and the difficulties that accompany ageing can lead to situations of loneliness and, in the worst cases, isolation. In cases of institutionalization, the attenuating effect of family support may increase when people are faced by the need to deal with a new life stage like old age. The goal of this study was to analyze the frequency and type of family contacts that are maintained in old people's residences, as well as the old people's degree of satisfaction with such contacts. A sample of 50 institutionalized people over 70 years of age completed the Cuestionario de Evaluación de Calidad de Vida en contexto Residencial (CECAVI-R, in Spanish, the Questionnaire to Assess Quality of Life within a Residential Context). The results show that the oldest people in the residences have more frequent family contacts. Taking gender into account, the frequency of family contacts varied as a function of the visitor (grandchildren, siblings, etc.). Lastly, the analysis of satisfaction with family relations was positive, and the younger inmates displayed the least satisfaction.

Key words: Social networks. Family. Older people. Institutionalization. Satisfaction.

Correspondencia: $\mathrm{M}^{\mathrm{a}}$ del Carmen Pérez Fuentes. Facultad de Psicología. Universidad de Almería. Ctra. de Sacramento, s/n. C.P. 04120. Almería (España). E-mail: $\underline{\text { mpf412@ual.es }}$ 
El estudio de la Calidad de Vida en los mayores viene cobrando una mayor preeminencia en los últimos años, debido fundamentalmente al fenómeno de envejecimiento poblacional, así como a la tendencia más actual de "dar vida a los años" (Leiva, 1995; Bayarre, Pérez y Menéndez, 2008). Así pues, en aras de proporcionar una atención integral al mayor, se tendrán en consideración todas aquellas dimensiones que van a determinar su Calidad de Vida. Dentro de la perspectiva multidimensional de Calidad de Vida, la esfera social ocupa un lugar importante como modulador de las circunstancias y/o sucesos que acontecen con la llegada de la vejez.

Dentro del área social, podemos diferenciar dos modalidades de apoyo: las relaciones sociales (amigos, vecinos, etc.), y las relaciones familiares; pudiendo ambas, de manera conjunta, facilitar el ajuste del individuo a las circunstancias que acontecen durante la vejez. Por un lado, el apoyo familiar funciona como un refuerzo para afrontar las crisis vitales del individuo; y por otro, el apoyo social se hace necesario en la medida en que va a facilitar la adaptación del sujeto a los cambios acontecidos en esta etapa de transiciones (Sáez, Meléndez y Aleixandre, 1994).

En un estudio realizado por Sáez, Meléndez y Aleixandre (1995), se pidió a una muestra de población mayor que identificaran y valorasen los problemas propios de su edad, observando como la soledad, se situaba en la primera posición, como la variable por la que más directamente se sentían afectados los participantes (59\%), seguido del rechazo familiar, con un $33 \%$. Además, encontraron diferencias en cuanto al género respecto a: la desadaptacion social, afectando de manera más intensa a hombres que a mujeres; y el tipo de apoyo social que demandan, decantándose los hombres hacia el apoyo social, mientras que las mujeres tienden a buscar el apoyo familiar en mayor medida. Así pues, dentro de las redes de apoyo que se dan en la vejez, no se ha de pasar por alto que las relaciones familiares suponen, como ya se apuntó anteriormente, un elemento primordial, en la determinación del bienestar y la Calidad de Vida del individuo (Barrón, 1996; Buendía, 1994; Montorio, 1994; Rodríguez, 1995).

En esta misma línea, Antonucci y Jackson (1990) se interesaron por el análisis diferencial del apoyo prestado por familiares y amigos. Lo que sugieren estos autores es, que el apoyo familiar, resulta decisivo durante los periodos de crisis, especialmente durante el curso de enfermedades crónicas. Así, un ejemplo de influencia del apoyo familiar en la salud lo encontramos en el estudio llevado a cabo por Redondo-Sendino, Guallar-Castillón, Banegas y Rodríguez-Artalejo (2005), en el cuál se demostró, que la frecuencia de contactos familiares mejoraba el conocimiento del estado hipertensivo en una muestra de ancianos con HTA.

Por el contrario, el apoyo prestado por amigos funciona más bien como refuerzo de la integración social del anciano. Para otros autores, como Yanguas, Leturia, Gutiérrez, Martín y Aragón (1993), el apoyo familiar implica, una percepción del nivel de apoyo mayor y de forma más clara, que cuando ésta proviene del círculo de amigos. 
Ante el incremento del número de personas mayores, y teniendo en consideración los cambios en la estructura de la unidad familiar acontecidos a lo largo de las últimas décadas (Gaymu, 2008; Pérez, 1999), podría verse variada la forma en que se ofertará el apoyo informal en la población mayor. Así, el estado civil va a determinar en gran medida los vínculos familiares con los que las personas cuentan durante la vejez. En Europa, más del $60 \%$ de los adultos mayores están casados, y una de cada dos mujeres de edad avanzada es viuda frente al 11.6\% de los hombres (Gaymu, 2008).

Con el fin de analizar las redes sociales de la población mayor, se llevaron a cabo una serie de trabajos a partir de fuentes de datos como la Encuesta sobre Salud, Envejecimiento y Jubilación en Europa (SHARE, 2004) o la Encuesta Social Europea (ESS, 2006). Estos análisis nos revelan que otro condicionante de la disponibilidad futura de familiares podría ser la separación o el divorcio. En nuestro país, el número de separados y divorciados que superan los 65 años se encuentra en torno al $1 \%$. Por otro lado, además de la pareja, los hijos serían una de las principales fuentes de apoyo con la llegada de la vejez de los progenitores. En España, el 13.5\% de los mayores no tienen hijos, un $14.3 \%$ dependen de un solo hijo, el $28.1 \%$ de dos y el $44 \%$ de más de dos hijos. Por otro lado, el género también puede marcar diferencias en la frecuencia con que se tiene contacto entre padres e hijos. En general, a cualquier edad las mujeres tienen un contacto más frecuente con sus hijos que los hombres (Lorenzo y Castejón, 2009).

La mayor parte de los individuos nacen, viven y mueren en un contexto familiar; aunque si bien es cierto que, con la llegada de la vejez, son muchos los que se ven en la tesitura de trasladarse a una institución geriátrica. No obstante, consideramos oportuno apuntar que la tendencia a generalizar la afirmacion de que todas las personas de edad avanzada terminan sus días en una residencia no es más que otro de los estereotipos que frecuentemente acompañan a la etapa de la vejez (Gázquez et al., 2009; Salvarezza, 1998).

Con el paso de los años, la probabilidad de experimentar pérdidas sociales irá en aumento, lo cual conlleva una alteración de la red de apoyo social del individuo (Romero, 2006), produciendo esto, a su vez, un deterioro de su Calidad de Vida (Winningham y Pike, 2007). Si centramos la atención en el contexto residencial y las modalidades de apoyo que tienen los mayores, observamos como la familia cobra especial relevancia en los procesos de adaptación e integración de la persona mayor durante su permanencia en el centro. El hecho de mantener vínculos de afecto con los hijos y los nietos, va a promover el intercambio intergeneracional y ofrecer a la persona mayor una sensación de seguridad ante la posibilidad de requerir su apoyo en situaciones críticas (Chappel, 1991; Gómez, López, Moya y Hernández, 2005; Lex, Hernández, Pereira y Sardiña, 2008).

En cuanto a la percepción de cercanía en las relaciones padres e hijos, ésta, puede calificarse como cercana, aunque en la mayoría de los casos serán los padres 
ancianos los que se refieran al trato con su prole como más estrecho (Hofffman, Paris y Hall, 1996). A otro nivel, aunque no menos importante, encontramos las relaciones entre hermanos. Connidis (1989) ya ensalzaba este tipo de apoyo fraternal, sobre todo cuando éste tenía lugar entre el género femenino. Posteriormente, otros autores aluden a la importancia de este tipo de apoyo entre hermanos, sobre todo al hecho de presentarse como la figura familiar con la que más tiempo comparten su vida (Craig, 1996; Papalia y Olds, 1997). En trabajos posteriores, se indica que, si bien es cierto que la relación fraternal se mantiene constante a lo largo de la vida, será en la vejez cuando tenga lugar una reactivación de la misma (Satir, 2002).

Los diferentes modos de contacto familiar que se producen desde el entorno residencial, se pueden agrupar en tres tipos: llamadas de teléfono, visitas de los familiares al centro y salida del residente al domicilio familiar. Las dos primeras, son las que se llevan a cabo en las dependencias de la residencia, además de ser las que se dan con mayor frecuencia, sobre todo entre la población femenina residente (Pérez, 2005).

El hecho de recibir llamadas o visitas de familiares, va a otorgar la sensación al mayor institucionalizado, de que los vínculos con el exterior no se han perdido, lo que a su vez infiere en un nexo de continuidad con la vida anterior al ingreso (Barenys, 1990). Según un estudio estatal sobre mayores en residencias realizado por el Instituto de Mayores y Servicios Sociales, en el año 2004, más del 50\% de los mayores que habitan en residencias reciben al menos una visita a la semana, frente a un $15 \%$ que no ha recibido ninguna a lo largo del último mes. Por otro lado, las personas de edades más avanzadas y las mujeres, son los grupos que reciben a familiares con mayor frecuencia en la residencia. Los datos obtenidos en esta misma encuesta, también hacen referencia a otra modalidad de relación del mayor con sus familiares desde la residencia, hablamos de los contactos telefónicos. Así pues, se puede observar cómo un $14.5 \%$ de los residentes con estancias de larga duración mantienen conversaciones telefónicas con familiares o amigos todos o casi todos los días, un $26.9 \%$ realizan llamadas dos o tres veces a la semana y un mayoritario $31.3 \%$ no realizó ni recibió llamadas en las tres últimas semanas. El grupo de residentes permanentes que con mayor frecuencia hace uso del teléfono, como modo de contactar con familiares desde la residencia, son las mujeres más jóvenes (Pérez, 2005).

En el momento en que la persona mayor institucionalizada no recibe, o no percibe, la suficiente atención por parte de los familiares, comienza a experimentar la sensación de pérdida de rol que éste desempeñaba en el núcleo familiar. Esto puede llevar asociado una serie de consecuencias, que pasan por una preocupación constante que puede derivar incluso en manifestaciones clínicas de índole psicológica o fisiológica (Pérez, 2005). Por tanto, los problemas familiares son considerados como un factor que va a influir de manera considerable en la estabilidad de la persona mayor, y en definitiva, en su nivel de autonomía y bienestar. Tanto es así que el desarraigo del mayor 
institucionalizado con respecto a las redes de apoyo familiar podría aumentar la probabilidad de padecer de ansiedad, depresión, psicomatización, introversión y aislamiento del entorno, entre otros (Guillén, 1998).

En relación con el grado de satisfacción que tienen los mayores institucionalizados con respecto a la relación que mantienen con sus familiares, Cava y Musitu (2000) llevaron a cabo un estudio en el que encontraron que este grupo parece tener una percepción más desfavorable del grado en que son valorados por su familia, si son comparados con otro grupo de mayores no institucionalizados. No obstante, estos autores no sostienen la consideración de la variable institucionalización como un indicador de bienestar en el mayor.

En un trabajo posterior, Rodríguez, La Rosa y Reyes (2006), pretendían valorar la interrelación de la familia con el adulto mayor institucionalizado. Para ello, aplicaron un cuestionario en el que se preguntaba a un grupo de 50 residentes por la frecuencia en los contactos con sus familiares, así como por el grado de satisfacción que tenían con respecto a dichas relaciones. Lo que encontraron fue que el $70 \%$ de los mayores entrevistados recibían visitas de familiares con una frecuencia mensual o cada dos o tres meses, siendo menor el grupo que afirmaba ser visitado semanalmente por la familia. La mayor parte de la muestra valoró las relaciones familiares como "buenas".

Más recientemente, en nuestro país, se llevó un estudio con el objetivo de medir y comparar el grado de satisfacción entre personas mayores institucionalizadas y no institucionalizadas. A partir de los datos obtenidos, se pudo llegar a concluir; por un lado, que la importancia otorgada a variables de relación social era más elevada en mayores institucionalizados; y por otro, que en este mismo grupo es donde se mantienen los porcentajes más elevados de insatisfacción hacia sus relaciones sociales (Molina, Meléndez y Navarro, 2008). A partir de estos resultados, estos autores, proponen como objetivo de intervención en el entorno residencial, la promoción de los contactos familiares con el fin de potenciar las redes de apoyo en la población mayor residente.

Así, el objetivo del presente trabajo es describir las relaciones familiares que mantienen un grupo de mayores institucionalizados, y valorar el grado de satisfacción que declaran con respecto a las mismas. Para ello, se atenderá a la inclusión de variables sociodemográficas como la edad y el género.

\section{METODO}

\section{Participantes}

El total de la muestra utilizada para el presente estudio es de 50 sujetos mayores de 70 años, todos ellos usuarios de Residencia de Ancianos de carácter público; con una media de edad de 81,26 años (DT=5,889). Siendo la edad mínima de los participantes de 72 años, y con una máxima de 94 años. De ellos, el 54\% son hombres y 
el $46 \%$ mujeres, con una media de edad de 80,78 (DT= 5,866) y 81,83 (DT= 5,997) años respectivamente. En cuanto al estado civil de la muestra, el 20\% están solteros, el 14\% casado, el $60 \%$ viudo, el $2 \%$ separado y el $4 \%$ forman pareja de hecho (Tabla1).

Tabla 1. Distribución de la muestra por género, estado civil y edad

\begin{tabular}{cccccccccccc}
\hline & \multicolumn{4}{c}{ Género } & \multicolumn{1}{c}{ Estado Civil } & \multicolumn{6}{c}{ Edad } \\
\cline { 2 - 13 } & Hombre & Mujer & Soltero & Casado & Viudo & Div/Sp & Pareja Hcho & $70-74$ & $75-79$ & $80-84$ & 85 o más \\
\hline $\mathrm{N}$ & 27 & 23 & 10 & 7 & 30 & 1 & 2 & 8 & 10 & 15 & 17 \\
$\%$ & $54 \%$ & $46 \%$ & $20 \%$ & $14 \%$ & $60 \%$ & $2 \%$ & $4 \%$ & $16 \%$ & $20 \%$ & $30 \%$ & $34 \%$ \\
\hline
\end{tabular}

\section{Instrumento}

El instrumento utilizado es un Cuestionario de Evaluación de Calidad de Vida en Contexto Residencial "CECAVI-R" (Molero, 2011), para población mayor de 65 años en contexto residencial, con aplicación mediante entrevista estructurada. El cuestionario está formado por un total de 56 ítems, organizados en 7 hojas de aplicación. Aunque el presente estudio forma parte de una investigación más amplia sobre Calidad de Vida, en este caso, se atenderá solamente a los datos recogidos en la hoja 3, que corresponde al área de "Relaciones sociales y familia" (13 ítems); y más concretamente a los ítems relacionados con las relaciones familiares (8 ítems).

El formato de respuesta es cerrado, mediante una escala de valor (1= nunca, nada, muy mala / 2= algunas veces, poco, mala / 3= a veces, normal, regular / $4=$ bastantes veces, bastante, buena $/ 5=$ muchas veces, siempre, mucho, muy buena). Además, en cada opción de respuesta se añade un valor adicional: $\mathrm{NP}=$ no es pertinente a su estado o situación real, o no contesta. El tiempo de aplicación del cuestionario completo es de 15-20 minutos de media, por sujeto. Aunque esto último irá en función de las características propias de cada participante.

\section{Procedimiento}

Antes de proceder a la implementación del cuestionario, se solicitaron los permisos pertinentes a la Dirección de la Residencia, donde se informaba del carácter anónimo en el tratamiento de los datos, así como de la finalidad de la investigación. Para la aplicación del cuestionario fueron instruidas dos personas que se desplazaron a la Residencia de Ancianos, situada a las afueras del casco urbano de Almería. En una reunión previa con los responsables de la institución, se acordó una selección de los sujetos que conformarían la muestra, por parte del Equipo Técnico; atendiendo a la disponibilidad de cada uno de los usuarios, con el fin de no entorpecer la rutina de los mismos. A cada participante se le ofreció una breve explicación del estudio y se le proporcionó una hoja de consentimiento informado, donde se garantizó la confidencialidad y el uso de la información para el estudio.

La aplicación del instrumento se realiza de manera individual, mediante entrevista personal. Tras una breve introducción de lo que se va a tratar, y recogida de 
datos personales del sujeto; se procede a la presentación de los ítems, con las aclaraciones pertinentes al inicio de cada hoja del cuestionario. Es decir, antes de proceder con la aplicación, se lleva a cabo una breve introducción que ayude al sujeto a situarse en la temática sobre aquello sobre lo que se le va a cuestionar.

\section{Diseño y análisis de datos}

Para el análisis de los datos se ha utilizado el programa estadístico SPSS 20. Se trata de un estudio descriptivo, de carácter transversal. Los parámetros descriptivos fueron realizados mediante el análisis de frecuencias y las tablas de contingencia teniendo en cuenta el género. Para la realización de los análisis de puntuaciones medias se aplicó la Prueba $t$ para muestras independientes, con el objetivo de observar si hay diferencias significativas en función del género, y la prueba ANOVA de un factor y las comparaciones múltiples o contrastes post hoc (Scheffé), para analizar las diferencias entre las medias en los distintos grupos de edad. Por último, para conocer si existe una relación entre la variable edad y los valores otorgados a los diferentes ítems del factor Relaciones sociales y familia utilizamos la Correlación de Pearson.

\section{RESULTADOS}

Respecto a las diferencias de género, tal y como podemos observar en la tabla 2, los hombres reciben visitas de sus familiares con una frecuencia significativamente superior a las mujeres. Por otra parte, al concretar la figura familiar que realiza las visitas, en el caso de los nietos, sería el género femenino el que destaca de manera significativa como el grupo que con mayor frecuencia recibe a sus nietos en la residencia. En cuanto al resto de los familiares que llevan a cabo las visitas (hijos y hermanos), no se encuentran diferencias significativas entre hombres y mujeres.

Atendiendo a otros ítems referentes a la satisfacción con la frecuencia de las visitas o, de forma más general, a la satisfacción con los contactos familiares, no se han encontrado diferencias significativas en función del género; observándose puntuaciones medias muy similar en ambos sexos con respecto a este último ítem.

Tabla 2. Relaciones familiares y género. Descriptivos y prueba $t$ para la igualdad de medias

\begin{tabular}{|c|c|c|c|c|c|c|c|c|}
\hline \multirow{3}{*}{ Ítems Relaciones Familiares } & \multicolumn{6}{|c|}{ Género } & \multirow{3}{*}{$t$} & \multirow{3}{*}{ Sig. } \\
\hline & \multicolumn{3}{|c|}{ Hombres } & \multicolumn{3}{|c|}{ Mujeres } & & \\
\hline & $\mathrm{N}$ & Media & DT & $\mathrm{N}$ & Media & DT & & \\
\hline Recibo vistas de familiares con una frecuencia & 27 & 3,85 & 1,199 & 23 & 3,04 & 1,331 & 2,259 &, $028 *$ \\
\hline $\begin{array}{l}\text { Recibo visitas de algunos familiares con menos frecuencia } \\
\text { de la que me gustaría }\end{array}$ & 24 & 3,17 &, 816 & 20 & 3,60 & ,995 & $-1,588$ &, 120 \\
\hline Las visitas que recibo tienen una duración & 24 & 3,92 & ,929 & 20 & 3,40 & 883 & 1,879 & 067 \\
\hline Mis hijos/as me visitan y/o me llaman & 18 & 4,00 & 1,188 & 14 & 4,07 & 1,385 &,- 157 & ,876 \\
\hline Mis nietos/as me visitan y/o me llaman & 15 & 2,53 & 1,302 & 14 & 3,79 & 1,251 & $-2,637$ & ,014* \\
\hline Mis hermanos/as me visitan y/o me llaman & 18 & 2,39 & 1,501 & 15 & 2,13 & 1,187 &, 546 &, 589 \\
\hline Soy consciente de problemas familiares que me preocupan & 27 & 1,74 & 1,095 & 23 & 2,13 & 1,604 &,- 986 & ,331 \\
\hline Estoy satisfecho/a con las relaciones familiares & 27 & 3,74 & ,903 & 23 & 3,78 & ,998 &,- 156 & 877 \\
\hline
\end{tabular}


En la Tabla 3, se analizan las diferencias, en función de la edad, respecto a las características que describen las relaciones familiares en residencia. Para ello, se ha realizado la prueba ANOVA de un factor, que nos revela la existencia de diferencias significativas entre los grupos de edad, y para determinar los grupos en los que se dan tales diferencias, se realizaron los contrastes post hoc, concretamente la prueba Scheffé.

Tabla 3. Relaciones familiares y edad. Descriptivos, ANOVA y post hoc (Scheffé)

\begin{tabular}{|c|c|c|c|c|c|c|c|c|c|}
\hline \multirow{2}{*}{$\begin{array}{l}\text { Ítems Relaciones } \\
\text { Familiares }\end{array}$} & \multirow{2}{*}{ Edad } & \multirow{2}{*}{$\mathrm{N}$} & \multirow{2}{*}{ Media } & \multirow{2}{*}{ DT } & \multicolumn{2}{|c|}{ ANOVA } & \multirow{2}{*}{$\begin{array}{l}\text { Diferencia de } \\
\text { medias }\end{array}$} & \multirow{2}{*}{$\begin{array}{l}\text { Diferencia } \\
\text { de medias }\end{array}$} & \multirow{2}{*}{$\begin{array}{l}\text { Diferencia } \\
\text { de medias }\end{array}$} \\
\hline & & & & & $F$ & Sig. & & & \\
\hline \multirow{4}{*}{$\begin{array}{lrr}\text { Recibo vistas } & \text { de } \\
\text { familiares } & \text { con } & \text { una } \\
\text { frecuencia } & & \end{array}$} & $70-74$ (g1) & 8 & 2,88 & 1,808 & \multirow{4}{*}{2,160} & \multirow{4}{*}{,106 } & \multirow{4}{*}{$\left|\begin{array}{l}\mathrm{g} 1-\mathrm{g} 2 \\
\mathrm{~g} 1-\mathrm{g} 3\end{array}\right|$} & \multirow{4}{*}{$\left|\begin{array}{l|l}\mathrm{g} 2-\mathrm{g} 3 & \mid \mathrm{g} 3-\mathrm{g} 4 \\
\mathrm{~g} 2-\mathrm{g} 4 & \mid \\
\mathrm{g} 1-\mathrm{g} 4\end{array}\right|$} & \\
\hline & $75-79(\mathrm{~g} 2)$ & 10 & 3,50 &, 707 & & & & & \\
\hline & $80-84(\mathrm{~g} 3)$ & 15 & 3,13 & 1,457 & & & & & \\
\hline & $>85(\mathrm{~g} 4)$ & 17 & 4,06 & 1,029 & & & & & \\
\hline \multirow{4}{*}{$\begin{array}{l}\text { Recibo visitas de } \\
\text { algunos familiares con } \\
\text { menos frecuencia de la } \\
\text { que me gustaría }\end{array}$} & $70-74$ (g1) & 5 & 3,80 &, 837 & \multirow{4}{*}{1,829} & \multirow{4}{*}{, 157} & \multirow{4}{*}{\begin{tabular}{|l|}
$\mathrm{g} 1-\mathrm{g} 2$ \\
$\mathrm{~g} 1-\mathrm{g} 3$
\end{tabular}} & \multirow{4}{*}{\begin{tabular}{|l||l|}
$\mathrm{g} 2-\mathrm{g} 3$ & $\mathrm{~g} 3-\mathrm{g} 4$ \\
$\mathrm{~g} 2-\mathrm{g} 4$ & $\mid$ \\
$\mathrm{g} 1-\mathrm{g} 4$
\end{tabular}} & \\
\hline & $75-79(\mathrm{~g} 2)$ & 10 & 3,70 & 675 & & & & & \\
\hline & $80-84$ (g3) & 12 & 3,42 & 1,165 & & & & & \\
\hline & $>85(\mathrm{~g} 4)$ & 17 & 3,00 &, 791 & & & & & \\
\hline \multirow{4}{*}{$\begin{array}{l}\text { Las visitas que recibo } \\
\text { tienen una duración }\end{array}$} & $70-74$ (g1) & 5 & 3,80 & 1,095 & \multirow{4}{*}{,202 } & \multirow{4}{*}{,894 } & & \multirow{4}{*}{\begin{tabular}{|l||l|}
$\mathrm{g} 2-\mathrm{g} 3$ & $\mathrm{~g} 3-\mathrm{g} 4$ \\
$\mathrm{~g} 2-\mathrm{g} 4$ & $\mid$ \\
$\mathrm{g} 1-\mathrm{g} 4$
\end{tabular}} & \\
\hline & $75-79(\mathrm{~g} 2)$ & 10 & 3,60 &, 843 & & & g1-g2 & & \\
\hline & $80-84$ (g3) & 12 & 3,83 & 835 & & & $|\mathrm{~g} 1-\mathrm{g} 3|$ & & \\
\hline & $>85(\mathrm{~g} 4)$ & 17 & 3,59 & 1,064 & & & & & \\
\hline \multirow{4}{*}{$\begin{array}{l}\text { Mis hijos/as me visitan } \\
\text { y/o me llaman }\end{array}$} & $70-74(\mathrm{~g} 1)$ & 6 & 3,33 & 1,862 & \multirow{4}{*}{, 884} & \multirow{4}{*}{,461 } & & \multirow{4}{*}{\begin{tabular}{|l||l|}
$\mathrm{g} 2-\mathrm{g} 3$ & $\mathrm{~g} 3-\mathrm{g} 4$ \\
$\mathrm{~g} 2-\mathrm{g} 4$ & $\mid$ \\
$\mathrm{g} 1-\mathrm{g} 4$
\end{tabular}} & \\
\hline & $75-79(\mathrm{~g} 2)$ & 4 & 4,50 &, 577 & & & g1-g2 & & \\
\hline & $80-84$ (g3) & 7 & 4,00 & 1,414 & & & $\mid$ & & \\
\hline & $>85(\mathrm{~g} 4)$ & 15 & 4,20 & 1,014 & & & & & \\
\hline & $70-74(\mathrm{~g} 1)$ & 4 & 1,00 &, 000 & & & & & \\
\hline & $75-79(\mathrm{~g} 2)$ & 4 & 3,00 & 1,155 & & & g1-g2 & g3-g4 & * \\
\hline visitan y/o me llaman & $80-84$ (g3) & 6 & 3,50 & 1,225 & 5,626 & ,004 & & $\mathrm{g} 2-\mathrm{g} 4|*| \mathrm{g} 1-\mathrm{g} 4 \mid$ & $* *$ \\
\hline & $>85(\mathrm{~g} 4)$ & 15 & 3,60 & 1,242 & & & & & \\
\hline & $70-74(\mathrm{~g} 1)$ & 5 & 2,40 & 1,517 & & & & & \\
\hline Mis hermanos/as me & $75-79(\mathrm{~g} 2)$ & 7 & 3,00 & 1,414 & & & $\mathrm{~g} 1-\mathrm{g} 2$ & g2-g3 & \\
\hline visitan y/o me llaman & $80-84(\mathrm{~g} 3)$ & 6 & 2,83 & 1,169 & 2,281 & ,100 & $\mid$ & \begin{tabular}{|l|l|l}
$\mathrm{g} 2-\mathrm{g} 4$ & $\mathrm{~g} 1-\mathrm{g} 4$
\end{tabular} & \\
\hline & $>85(\mathrm{~g} 4)$ & 15 & 1,67 & 1,175 & & & & & \\
\hline & $70-74(\mathrm{~g} 1)$ & 8 & 2,25 & 1,389 & & & & & \\
\hline Soy consciente de & $75-79(\mathrm{~g} 2)$ & 10 & 1,60 & 1,265 & 1536 & 218 & g1-g2 & g2-g3 & \\
\hline $\begin{array}{l}\text { problemas familiares } \\
\text { gue me preocupan }\end{array}$ & $80-84$ (g3) & 15 & 1,47 & 1,060 & 1,536 & ,218 & $|\mathrm{g} 1-\mathrm{g} 3|$ & $|\mathrm{g} 2-\mathrm{g} 4|$ & \\
\hline & $>85(\mathrm{~g} 4)$ & 17 & 2,35 & 1,539 & & & & & \\
\hline & $70-74(\mathrm{~g} 1)$ & 8 & 2,75 &, 707 & & & & & \\
\hline Estoy satisfecho/a con & $75-79(\mathrm{~g} 2)$ & 10 & 4,10 &, 568 & & & | g1-g2 & |g2-g3 & \\
\hline $\begin{array}{ll}\text { las } & \text { relaciones } \\
\text { familiares } & \end{array}$ & $80-84$ (g3) & 15 & 3,93 &, 884 & 4,643 & ,006 & g1-g3 & $|\mathrm{g} 2-\mathrm{g} 4|$ & $*$ \\
\hline & $>85(\mathrm{~g} 4)$ & 17 & 3,88 & ,993 & & & & & \\
\hline
\end{tabular}

En primer lugar, haciendo referencia a la frecuencia de las visitas de los nietos/as, se observan diferencias significativas del grupo de mayores de 85 años respecto a los demás grupos, siendo estos los que son visitados más frecuentemente por sus nietos, seguidos de los grupos 80-84 y 75-79 años, y el grupo de residentes más jóvenes (70-74 años). Por otro lado, en la cuestión relativa a satisfacción general con las relaciones familiares, se obtienen diferencias significativas entre todos los grupos de edad. Así, los más satisfechos con las relaciones que mantienen con su familia son los residentes de entre 75-79 años, seguidos por los grupos 80-84 y 85 años en adelante; por último, encontramos que los de edades inferiores (70-74 años) son quienes muestran un menor grado de satisfacción hacia las relaciones familiares que mantienen desde la residencia. 
En cuanto al resto de los ítems, no se encuentran diferencias significativas entre los grupos etarios, con respecto a la frecuencia general y en otros contactos familiares (hijos o hermanos), o nivel de conocimiento de la problemática familiar que tiene el residente.

Para el análisis de la correlación entre la edad y los distintos ítems sobre las relaciones familiares (frecuencia, contactos, satisfacción,...), se toman las edades sin agrupar, por lo que empleamos el coeficiente de correlación de Pearson. De esta forma, tal y como se puede ver en la Tabla 4, se dan correlaciones entre la variable edad y algunos de los aspectos que hacen referencia a las características de las relaciones que mantienen los mayores institucionalizados con sus familiares. En primer lugar, observamos una correlación positiva entre la edad y la frecuencia de las visitas de los nietos/as, es decir, a mayor edad, mayor es también la frecuencia con la que los nietos visitan a sus abuelos en residencia. En segundo lugar, podemos comprobar cómo, en el caso de ser los hermanos/as los que realizan las visitas, se da una correlación negativa con respecto a la variable edad; por lo que, en este caso, a medida en que aumenta la edad de los residentes, son visitados por sus hermanos con menor frecuencia.

Tabla 4. Correlación: edad y relaciones familiares

\begin{tabular}{lccc}
\hline \multicolumn{1}{c}{ Relaciones Familiares } & $\mathrm{N}$ & Pearson & Sig. (bilateral) \\
\hline Recibo visitas de familiares con frecuencia & 50 &, 158 &, 274 \\
Recibo visitas de algunos familiares con menos frecuencia de la que me gustaría & 44 &,- 297 &, 050 \\
Las visitas que recibo tienen una duración & 44 &,- 092 &, 554 \\
Mis hijos/as me visitan y/o me llaman & 32 &, 217 &, 233 \\
Mis nietos/as me visitan y/o me llaman & 29 &, 442 &, $016^{*}$ \\
Mis hermanos/as me visitan y/o me llaman & 33 &,- 392 &, $024^{*}$ \\
Soy consciente de problemas familiares que me preocupan & 50 &, 026 &, 859 \\
Estoy satisfecho con las relaciones familiares & 50 &, 167 &, 247 \\
\hline
\end{tabular}

\section{DISCUSION}

Con respecto al género se han detectado diferencias significativas en la frecuencia de las visitas familiares en general, resultando más frecuentes en hombres que en mujeres. Este resultado no concuerda con los datos presentados por el IMSERSO (Pérez, 2005), donde son las mujeres de edades más avanzadas las que reciben a los familiares con mayor frecuencia en la residencia. No obstante, teniendo en cuenta que, en este caso, nos basamos en la frecuencia subjetiva de las visitas, es decir, lo que perciben y lo que declaran los sujetos entrevistados, se debería atender a otras cuestiones que, aún no mostrándose significativas en la presente investigación, podrían influir en la percepción de la frecuencia en los contactos. Hacemos referencia, por ejemplo, a una mayor conciencia de los problemas familiares en las mujeres que en los hombres, y por tanto, quizás por la necesidad de éstas, de pasar más tiempo con los familiares (Lorenzo y Castejón, 2009). A este respecto no es de obviar que existe una tendencia de las 
mujeres a buscar el apoyo familiar, en mayor medida que en caso de los varones (Sáez et al., 1995), lo que, por otra parte, podría estar condicionando el umbral de lo que éstas consideran una frecuencia aceptable en los contactos con sus familiares.

En cuanto a la edad, se obtienen resultados que apoyan la idea general de que los grupos de edades avanzadas serían los más visitados por familiares en residencia (Pérez, 2005). Al concretar la figura del que visita, se obtienen puntuaciones que difieren en función de la edad del residente. Así, tenemos que la frecuencia de las visitas de los nietos aumenta a medida que también lo hace la edad del residente, lo que pudiera estar explicado por una mayor probabilidad, a edades avanzadas, de que los nietos/as hayan alcanzado la adolescencia y, en muchos casos la adultez; lo que facilita los contactos de manera más autónoma.

Por otro lado, observábamos cómo ocurre el efecto contrario en el caso de las visitas por parte de los hermanos/as, viendo éstas su frecuencia disminuida a medida en que los residentes avanzan en edad. Estos resultados no casan con la idea de que en la vejez tendría lugar una reactivación en la frecuencia e intensidad de las relaciones fraternales (Satir, 2002), pudiendo estar explicado a partir de la influencia que la edad ejerce sobre la disponibilidad de estos familiares (Craig, 1996; Papalia y Olds, 1997). Es decir, teniendo presente que entre hermanos no suele darse demasiada diferencia de edad, es fácil que tengan la misma o similar a la del residente, por lo que al aumentar la edad, aumentará también el riesgo de pérdida por defunción (Winningham y Pike, 2007).

Por último, el hecho de que los niveles más bajos de satisfacción con las relaciones familiares en residencia, se sitúen en el grupo de residentes más jóvenes, nos lleva a repasar algunos de los resultados anteriores: dentro del entorno residencial, a edades más inferiores se da una menor frecuencia en los contactos familiares, lo que puede hacer más difícil la adaptación del sujeto a situaciones críticas (Antonucci y Jackson, 1990; Guillén, 1998) y, por tanto, una percepción más desfavorable de las circunstancias y el entorno residencial. A todo lo anterior añadimos la idea de que son los mayores institucionalizados los que mayor porcentaje de insatisfacción presentan, quizás por el hecho de que también son éstos los que consideran los contactos con sus familiares de mayor valía (Molina et al., 2008). Esto, los puede hacer más exigentes en sus valoraciones, sobre todo en edades más tempranas, en las que todavía no se han desvinculado totalmente de su vida anterior al ingreso.

La necesidad de conocer cómo son las relaciones que mantienen las personas mayores (institucionalizadas o no) con sus familiares, surge a partir de la idea, de que un mejor conocimiento de esta esfera social, nos permitirá plantear una serie de intervenciones con la población mayor, en este caso institucionalizada, para la mejora de su Calidad de Vida. De hecho, hay estudios de los que se extraen datos que apoyan el papel determinante que cumple el apoyo social, en general, y en mayor medida, las relaciones familiares; en la capacidad funcional (Tabio, Rubido y López, 2011), salud 
física (Fernández, 1998) o mental (Read y Grundy, 2011). Respecto a esta última, encontramos trabajos donde se analiza la relación que existe entre la falta de apoyo familiar y el desarrollo de síntomas depresivos en adultos mayores (Cerquera y Meléndez, 2010), actuando las relaciones con la familia, cuando éstas son positivas, como un moderador de la citada sintomatología (Zhang y Li, 2011). Es tal el grado de determinación que las redes familiares tienen sobre el bienestar del mayor, que recientemente se propone que una mala comunicación del anciano con sus familiares, a pesar de tenerlos cerca, podría llegar a aumentar, en conjunción con otros factores, el riesgo de suicidio, ocupando la disfunción familiar el primer lugar entre los factores de riesgo de suicidio en la población mayor (Jiménez, 2011; Mellgvist et al., 2011).

Los mayores que viven en residencias parecen disponer de redes de apoyo familiar suficientes, aunque con contactos limitados en su frecuencia. Respecto a esto, hay que tener en cuenta aspectos relacionados que pudieran estar dificultando la periodicidad en los contactos familiares, como pueden ser los cambios en la estructura familiar acontecidos en los últimos años (Abellán, Esparza y Pérez, 2011), o la ubicación de las residencias de alta ocupación que, con frecuencia suelen ser construidas en el retiro de núcleos urbanos (Pino, Crespo y Portela, 2010).

Por otro lado, características individuales como la edad o el género del residente, se ha podido observar cómo, en cierto modo, marcan ciertas diferencias en el modo de relacionarse con sus familiares; aunque para poder determinar hasta qué punto la institucionalización funciona como una variable de influencia en los modos de relacionarse del anciano con sus familiares sería necesario, en primer lugar, ampliar la muestra de estudio y, en segundo lugar, incluir una población mayor de similares características, no institucionalizados. Otro aspecto a contemplar como objeto de estudio sería la inclusión de la variable estado civil, la cual no ha sido considerada en el presente trabajo debido a la limitación de una muestra reducida y, por tanto, la imposibilidad de llevar a cabo un análisis completo de los datos.

En definitiva, podemos afirmar que en un entorno residencial la importancia que se le otorga a la familia por parte de las personas mayores que allí residen podría ser superior que en otros entornos de convivencia comunitaria. Por lo que, en consonancia con la propuesta de Molina et al. (2008), una intervención dirigida a la mejora de la Calidad de Vida en este contexto residencial, pasaría ineludiblemente por la promoción de los contactos familiares periódicos de la población mayor que allí reside, tanto en las dependencias del centro como en el exterior, cuando esto último fuera posible.

\section{REFERENCIAS}

Abellán, A., Esparza, C. y Pérez, J. (2011). Evolución y estructura de la población en situación de dependencia. Cuadernos de Relaciones Laborales, 29(1), 93-123. 
Antonucci, J.R. y Jackson, J.S. (1990). Apoyo social, eficacia interpersonal y salud: una perspectiva del transcurso de la vida. En L. Carstensen y B.A. Edelstein (Eds.), Gerontología clínica. Intervención psicológica y social. Barcelona: Martínez Roca.

Barenys, M.P. (1990). Residencias de ancianos. Análisis sociológico. Barcelona: Fundación La Caixa.

Barrón, A. (1996). Apoyo social. Aspectos teóricos y aplicaciones. Madrid: Siglo XXI.

Bayarre, H.D., Pérez, J. y Menéndez, J. (2008). Las transiciones demográfica y epidemiológica y la Calidad de Vida objetiva en la tercera edad. Geroinfo, 1(3).

Buendía, J. (1994). Envejecimiento y psicología de la salud. Madrid: Siglo XXI.

Cava, M.J. y Musitu, G. (2000). Bienestar psicosocial en ancianos institucionalizados y no institucionalizados. Revista Multidisciplinar de Gerontología, 10(4), 215-221.

Cerquera, A.M. y Meléndez, C. (2010). Factores socioculturales asociados a las características diferenciales de la depresión a través del envejecimiento. Pensamiento Psicológico, 14(7), 63-72.

Chappell, N.L. (1991). The role of family and friends in quality of life. Los Angeles: Academic Press.

Connidis, I. (1989). Family ties and aging. Toronto: Butterworths.

Craig, G. (1996). Desarrollo psicológico. México: Prentice Hall Hispanoamericana.

European Social Survey (2006). Data file edition 3.3. Norway: Norwegian Social Science Data Services.

Fernández, A. (1998). Apoyo social y Calidad de Vida en personas mayores. Madrid: Imprenta Serrano.

Gaymu, J. (2008). What family support will dependent elders have in 2030? European projections. Population \& Societies, 444, 1-4.

Gázquez, J.J., Pérez-Fuentes, M.C., Fernández, M., González, L., Ruiz, I. y Díaz, A. (2009). Estereotipos sobre la vejez y su relación con la formación en gerontología: un estudio intergeneracional. European Journal of Education and Psychology, 2(3), 263-273.

Gómez, M., López, A., Moya, M. y Hernández, O.R. (2005). Influencia de algunas variables en las relaciones familiares del adulto mayor. Revista Cubana de Medicina General Integral, 21, 1-12.

Guillén, F. (1998). Atención a personas mayores que viven en residencias: Protocolos de intervención psicosocial y sanitaria. En: Ministerio del trabajo y asuntos sociales IMSERSO. Los problemas familiares (p. 196-208). Madrid: IMSERSO.

Hoffman, L., Paris, S. y Hall, E. (1996). Psicología del desarrollo. Madrid: McGraw-Hill.

Jiménez, E. (2011). Psychosocial characterization of aged people who committed suicide. Medisan, 15(3), 339-345.

Leiva, F. (1995). La Calidad de Vida como método de valoración de las actuaciones en atención primaria. Atención Primaria, 16(1), 130-137.

Lex, E.I., Hernández, R., Pereira, I. y Sardiña, O. (2008). Caracterización de la atención familiar al adulto mayor. $A M C, 12(6)$.

Lorenzo, L. y Castejón, P. (2009). Formas de convivencia, relaciones personales y la experiencia de envejecer. En R. Díaz (coord.), Las Personas Mayores en España. Datos Estadísticos Estatales y por Comunidades Autónomas. Informe 2008. Tomo 1 (pp. 223-268). Madrid: IMSERSO.

Mellgvist, M., Wiktorsson, S., Joas, E. Ostling, S., Skooq, I. y Waern, M. (2011). Sense of coherence in elderly suicide attempters: the impact of social and health-related factors. International Psychogeriatrics, 1, 1-8.

Molero, M.M. (2011). Evaluación de la Calidad de Vida en mayores de 65 años en contexto residencial. Tesis Doctoral no publicada. Universidad de Almería, Almería, España. 
Molina, C., Meléndez, J.C. y Navarro, E. (2008). Bienestar y Calidad de Vida en ancianos institucionalizados y no institucionalizados. Anales de Psicología, 24, 312-319.

Montorio, I. (1994). La persona mayor. Guía aplicada de evaluación psicológica. Ministerio de asuntos sociales. Madrid: Instituto Nacional de Servicios Sociales.

Papalia, D. y Olds, S. (1997). Desarrollo humano. Bogotá: McGraw-Hill.

Pérez, C. (1999). La familia en su etapa de formación. Revista Cubana Medicina General Integral, 15(3), 237-40.

Pérez, L. (2005). Mayores en residencias. En M. Sancho (coord.) Las Personas Mayores en España. Informe 2004. Datos Estadísticos Estatales y por Comunidades Autónomas (p.811-907). Madrid: IMSERSO.

Pino, M.R., Crespo, J.M. y Portela, J. (2010). Descripción de los elementos espaciales en residencias de ancianos. Estudio en el noroeste de España. Revista de Investigación en Educación, 7, 61-71.

Ramos, F. (2002). Salud y Calidad de Vida en las personas mayores. Tabanque Revista Pedagógica, 16, 83-104.

Read, S. y Grundy, E. (2011). Mental health among older married couples: the role of gender and family life. Society of Psychiatry and Psychiatrical Epidemiology, 46, 331-341.

Redondo-Sendino, A., Guallar-Castillón, P., Banegas, J.R. y Rodríguez-Artalejo, F. (2005). Relación entre la red social y la hipertensión arterial en los ancianos españoles. Revista Española de Cardiología, 58(11), 1294-1301.

Rodríguez, M., La Rosa, M. y Reyes, M. (2006). Interrelación de la familia en el adulto mayor institucionalizado. Revista Cubana de Enfermería, 22(1).

Rodríguez, M.J. (1995). Psicología Social de la Salud. Madrid: Editorial Síntesis.

Romero, J. (2006). La fragilidad como punto de corte en Geriatría. Geriátrica, 22(2), 42-6.

Sáez, N., Meléndez, J.C. y Aleixandre, M. (1994). Los estereotipos en los ancianos: un estudio empírico y sus resultados. Revista de Psicología de la Educación, 14, 75-89.

Sáez, N.; Aleixandre, M. y Meléndez, J.C. (1995). Los problemas de la tercera edad según la tercera edad. Geriatrika, 11, 479-502.

Salvarezza, L. (1998). La vejez: una mirada gerontológica actual. México: Paidós.

Satir, V. (2002). Nuevas relaciones humanas en el núcleo familiar. México: Editorial Pax.

SHARE (2004). Survey of Health, Ageing and Retirement in Europe. En A. Börsch-Supan, A. Brugiavini, H. Jürges, J. Mackenbach, J. Siegrist, y G. Weber (eds.), First results from the Survey of Health, Ageing and Retirement in Europe. Mannheim: Instituto de Investigación de la Economía del Envejecimiento de Mannheim.

Tabio, Y.A., Rubido, D. y López, M.E. (2011). Factores de riesgo social en el adulto mayor. Revista Infociencia, 15(3), 1-11.

Winningham, R.G. y Pike, N.L. (2007). A cognitive intervention to enhance institutionalized older adults' social support networks and decrease loneliness. Aging Mental Health, 11, 716721.

Yanguas, J.J., Leturia, F.J., Gutiérrez, B., Martín, I. y Aragón, I. (1993). Las relaciones interpersonales y familiares: un modelo de trabajo con familias en residencias de ancianos. En J.J. Yanguas (coord.), Gerontología social: perspectivas teóricas y de intervención (p. 501-516). Jaén: Clave.

Zhang, B. y Li, J. (2011). Gender and marital status differences in depressive symptoms among elderly adults: the roles of family support and friend support. Aging and Mental Health, 15(7), 844-854.

Recibido: 1 de julio de 2011

Recepción Modificaciones: 11 de septiembre de 2011

Aceptado: 15 de octubre de 2011 